\title{
Daño cardíaco provocado por albendazol en fetos de ratones
}

\author{
Mendoza, C. ; Méndez, G. ${ }^{3}$; Duno, F. ${ }^{3}$; Sanabria, M.2 ; Matheus, N. ${ }^{1}$. \\ ${ }^{1}$ Departamento de Ciencias Básicas, ${ }^{2}$ Departamento de Medicina y Cirugia, Facultad de Ciencias Veterinarias, \\ Universidad Centroccidental "Lisandro Alvarado", Ap. Postal 267, Barquisimeto 3001, Venezuela, Tel/fax \\ (251)2592404. ${ }^{3}$ Unidad Educativa Colegio Francisco L. Martí, Barquisimeto, Estado Lara, Venezuela. \\ E-mail: nyurkym@ucla.edu.ve
}

\begin{abstract}
Resumen
Mendoza, C.; Méndez, G.; Duno, F.; Sanabria, M; Matheus, N.: Daño cardíaco provocado por albendazol en fetos de ratones. Rev. vet. 26: 1, 17-21, 2015. Albendazol es un antiparasitario efectivo contra protozoos y helmintos del tubo digestivo, cuyo empleo puede causar problemas gastrointestinales y efectos teratogénicos. El objetivo de esta investigación fue evaluar sus efectos sobre el desarrollo fetal del corazón de ratones. Se emplearon 10 ratones hembras NMRI, con peso promedio de $40 \mathrm{~g}$. Se conformaron dos grupos de 5 ejemplares cada uno (controles y experimentales). Una vez preñadas, las hembras del grupo experimental recibieron albendazol (vía oral, $5 \mathrm{mg} / \mathrm{kg}$ de peso vivo), a los 9, 10 y 11 días de gestación. El peso corporal fue monitoreado cada 3 días. Al nacer, las crías fueron sacrificadas y sus corazones se disecaron para efectuar estudios morfométricos, morfológicos e histopatológicos. Los resultados no revelaron diferencias estadísticas entre las ganancias diarias de peso de las madres de cada grupo. El número de crías fue significativamente mayor para el grupo de madres que recibieron albendazol, pero el peso de las mismas fue menor. Asimismo, se evidenció que ambas dimensiones cardíacas (transversal y longitudinal) fueron mayores en las crías de las madres no tratadas ( $3 \times 4 \mathrm{~mm}$ versus $2 \times 3 \mathrm{~mm}$ en las tratadas, $\mathrm{p} \leq 0,05)$, lo cual guardó relación con los promedios del volumen cardíaco de cada grupo $(\mathrm{p} \leq 0,01)$. En el grupo experimental, dos fetos nacieron muertos y en dos casos revelaron agenesia del rabo, así como aspecto redondeado en lugar de la forma cónica normal del corazón. El análisis histopatológico reveló degeneración mixoide de las fibras miocárdicas de los fetos del grupo experimental. Los resultados demuestran que el fármaco evaluado es capaz de causar daños teratogénicos en fetos de ratones, dato que debe alertar respecto a su peligrosidad durante la gestación de otras especies.
\end{abstract}

Palabras clave: ratón, gestación, albendazol, teratogénesis, degeneración cardíaca.

\begin{abstract}
Mendoza, C.; Méndez, G.; Duno, F.; Sanabria, M; Matheus, N.: Heart damage caused by albendazol in mice fetuses. Rev. vet. 26: 1, 17-21, 2015. Intestinal parasitic diseases are disorders caused by protozoa and helminths. These diseases lead to nutritional deterioration because parasites cause nutrient scavenging from hosts. For parasite control drugs such as albendazole are used. Short-term adverse effects include gastrointestinal alterations while longterm use has been associated with teratogenic effects. The objective of this research was to evaluate the effects of albendazole on mice fetal heart development. Ten NMRI female mice with an average weight of $40 \mathrm{~g}$ were selected. They were divided into two groups (control and experimental) of 5 animals each. After pregnancy was detected the experimental group was given albendazole ( $5 \mathrm{mg} / \mathrm{kg}$ liveweight) orally at 9,10 and 11 days of gestation. Body weight was monitored every three days. At birth the pups were sacrificed, the heart was dissected and morphometric, morphological and histopathological analysis were performed. No statistical difference was found between the average daily gain of mothers in each group. The number of offspring was higher for the group that received albendazole, but individual weight was lower. It also became clear that hearts' width and length were higher for the offspring of untreated compared to treated mothers ( $3 \times 4 \mathrm{~mm}$ and $2 \times 3 \mathrm{~mm}$ respectively, $\mathrm{p} \leq 0.05$ ), in correspondence to the considered average cardiac volume $(\mathrm{p} \leq 0.01)$. Histopathological analysis revealed myxoid degeneration of myocardial fibers of fetuses in the experimental group. These results demonstrate that albendazole caused teratogenic effects in mice pups, aspect that should be considered regarding the possible risks of its use during gestation in other species.
\end{abstract}

Key words: mouse, pregnancy, albendazole, fetal teratogeny, heart degeneration. 


\section{INTRODUCCIÓN}

Las parasitosis intestinales presentan una distribución mundial con alta prevalencia en las regiones tropicales y subtropicales ${ }^{20}$. Afectan tanto a seres humanos como animales, provocando cuadros digestivos que repercuten negativamente sobre el crecimiento y desarro1 lo ${ }^{18}$, a pesar del incremento de recursos terapéuticos eficaces y del establecimiento de programas de control ${ }^{20,21}$. Ello afecta el desarrollo económico y genera pobreza en los sectores sociales más desamparados ${ }^{4-6}$. Cada año se reportan entre 3.000 y 65.000 muertes por geohelmintiasis y 100.000 por amebiasis ${ }^{6,19,21}$.

Entre los antihelmínticos más utilizados figuran los benzimidazoles de larga vida media como oxfendazol, fenbendazol, albendazol y sus profármacos ${ }^{8}$, debido a que sus concentraciones eficaces se mantienen durante un período prolongado en plasma e intestino, lo que aumenta su efecto contra larvas y adultos inmaduros ${ }^{20,21}$. Albendazol es un benzimidazol rápidamente metabolizable y su principal metabolito, el sulfóxido de albendazol, también denominado ricobendazol, es un potente antihelmíntico utilizado extensamente tanto en animales como personas ${ }^{8}$. Tales antiparasitarios son capaces de provocar dismorfogénesis en especies como ovinos ${ }^{11}$, vacunos ${ }^{2}$ y ratas ${ }^{19}$. La actividad teratogénica del albendazol es atribuida a su transformación en sulfóxido, que alcanza mayores concentraciones sistémicas ${ }^{3}$.

A pesar que los benzimidazoles han sido reportados como teratogénicos, continúan siendo tratamientos de elección para el control del parasitismo intestinal. Por ello, la presente investigación tuvo como objetivo profundizar los conocimientos sobre los efectos nocivos del albendazol sobre el desarrollo del corazón fetal.

\section{MATERIAL Y MÉTODOS}

Se utilizaron 10 ratones hembras y 10 ratones machos no consanguíneos, de la cepa NMRI (Nacional Medicine Research Institute), con un peso promedio de $40 \mathrm{~g}$ y edad de 49 días, alojados en jaulas individuales y mantenidos bajo condiciones ambientales estables, con agua y alimento ad-libitum. El peso corporal se monitoreó por pesaje cada 3 días. Luego de una semana de aclimatación se introdujo un macho en cada jaula de las hembras durante dos días, para el apareamiento. El último día del apareamiento fue considerado como el día uno de preñez, previa verificación de la presencia de espermatozoides en el frotis vaginal.

Cinco hembras operaron como controles y las 5 restantes (experimentales), fueron dosificadas con albendazol (5 mg/kg de peso, vía oral), a los 9, 10 y 11 días de gestación. Al momento del parto se realizó el pesaje de la camada. La eutanasia se realizó por inhalación de éter, tras lo cual se disecaron los corazones, se evaluaron sus características macroscópicas y se realizaron estudios morfométricos, morfológicos e histopatológi- cos. El estudio fue conducido de acuerdo al Código de Bioética y Bioseguridad de FONACIT-Venezuela, 2009.

Los corazones se pesaron en una balanza analítica, registrándose también el color, consistencia, dimensiones $(\mathrm{mm})$ y volumen $\left(\mathrm{mm}^{3}\right)$ de cada uno. Las dimensiones se determinaron utilizando una regla milimetrada y el volumen se comprobó por el desplazamiento de agua destilada en una probeta graduada. Una porción del corazón fue fijada en formol tamponado al 10\% para los estudios histopatológicos. Mediante un micrótomo Jung AG (Heidelberg, Alemania) se obtuvieron secciones de $4 \mu \mathrm{m}$. Estos cortes fueron teñidos con hematoxilina-eosina de acuerdo a la técnica convencional.

Los resultados fueron analizados por el paquete estadístico SPSS, versión 17.0 para Windows. Se realizó la estadística descriptiva de las variables. Los resultados se expresaron como media \pm error estándar (EE) de los valores. La comparación de los valores medios de cada una de las mediciones entre los grupos experimental y control se realizó a través de una prueba " $t$ " de Student $(\mathrm{p}<0,05)$.

\section{RESULTADOS}

Peso materno. El peso corporal de las hembras preñadas fue expresado en ganancia diaria de peso (GDP) y obró como indicador del estado de salud de los roedores tratados con albendazol en la mitad de la gestación (9, 10 y 11 días de preñez). La GDP fue de $25 \mathrm{~g}$ /día tanto en las hembras tratadas como no tratadas.

Número y peso de las crías. De las 5 camadas obtenidas en cada grupo, se cuantificó un promedio de 8,6 crías para el grupo control y 11,0 crías para el grupo experimental, diferencia que fue estadísticamente significativa $(p<0,05)$. Es importante señalar que a pesar de que las camadas de las madres tratadas fueron más numerosas que la del grupo testigo, el peso promedio de las crías de este último grupo fue estadísticamente menor $(1,9 \mathrm{~g}$ versus $1,5 \mathrm{~g}$ respectivamente, $\mathrm{p}<0,05)$.

Estudios morfológico y morfométrico. Para conocer si el tratamiento con albendazol afectó el desarrollo fetal cardíaco se cuantificaron las dimensiones (transversales y longitudinales) del corazón de las crías provenientes de los animales tratados y se compararon con los no tratados. Se encontró que los promedios de la longitud y ancho del corazón fueron estadísticamente mayores $(p \leq 0,05$ y $p \leq 0,01)$ en animales provenientes de madres no tratadas (Figura 1). Asimismo, se evidenció que los corazones de los no tratados tenían un aspecto cónico mientras que los corazones de los tratados eran redondeados.

Con el fin de ratificar si efectivamente existía diferencia en el tamaño del corazón entre los grupos bajo estudio, se cuantificó el volumen cardiaco de cada cría (Figura 2) hallándose una disminución estadísticamente significativa en las crías de las madres tratadas en comparación con las provenientes de las no tratadas 


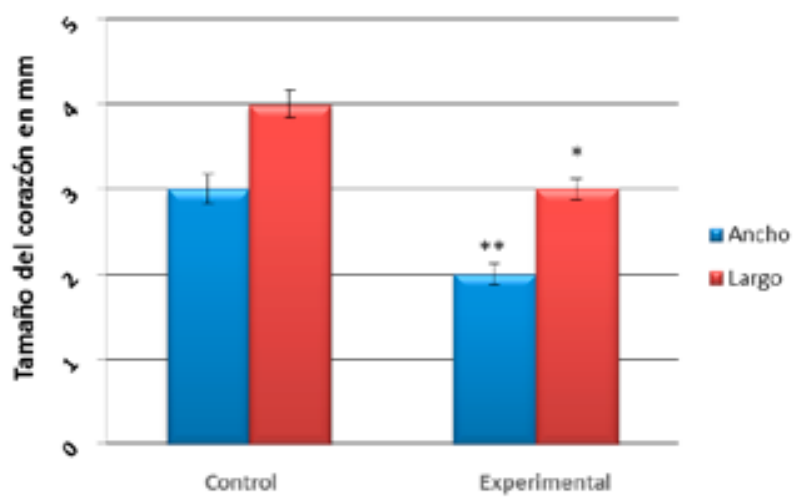

Figura 1. Efecto del tratamiento con albendazol sobre el tamaño de los corazones de las crías de madres experimentales comparados con los testigos (media aritmética \pm EE de 98 recién nacidos). Asteriscos indican diferencias entre tratados y controles $(* \mathrm{p}<0,05$; $* * \mathrm{p}<0,01)$.

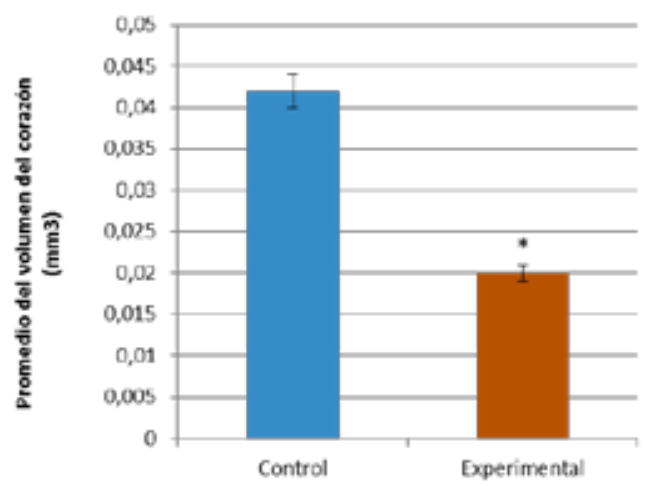

Figura 2. Efecto del tratamiento con albendazol sobre el volumen de los corazones de las crías de madres controles versus experimentales (media aritmética \pm EE de 98 recién nacidos). Asterisco indica diferencia entre tratados y controles $(* \mathrm{p}<0,01)$.
(0,02 versus $\left.0,04 \mathrm{~mm}^{3}, \mathrm{p} \leq 0,01\right)$. Tales cambios fueron congruentes con los tamaños de los respectivos grupos.

Es importante señalar que de las crías nacidas de las hembras tratadas con albendazol, dos de ellas presentaron agenesia de rabo y otras dos nacieron muertas.

Estudio histopatológico. La Figura 3 muestra cortes histológicos del miocardio de crías controles y experimentales. En los controles puede verificarse la presencia de tejido mixoide, miocardiocitos inmaduros y algunos adipocitos, hallazgos normales en recién nacidos de esta especie. Por el contrario, en las crías del grupo experimental, además de áreas de tejido laxo de tipo mixoide e inmadurez de miocardiocitos y adipocitos infiltrados entre las fibras musculares, se evidenció degeneración mixoide del septum interventricular y de algunas zonas de las paredes de las válvulas atrioventriculares derechas e izquierdas, así como leve engrosamiento de dicho septum.

\section{DISCUSIÓN}

Los antihelmínticos más efectivos del grupo de los benzimidazoles son los que tienen la vida media más larga, como el albendazol 12,15, los cuales han demostrado ser teratogénicos en ratas, ratones, conejos, porcinos, ovinos y vacunos, ocasionando malformaciones esqueléticas ${ }^{1,3,9}$.

Los resultados de este estudio indican que la administración de albendazol no afectó el peso materno cuantificado como ganancia diaria de peso. Asimismo se constató que el promedio del número de crías por camada de las hembras tratadas fue mayor que el número de crías provenientes de las hembras no tratadas $(11,0 \mathrm{y}$ 8,6 crías, respectivamente), sin embargo es importante resaltar que aunque el número de crías de las hembras
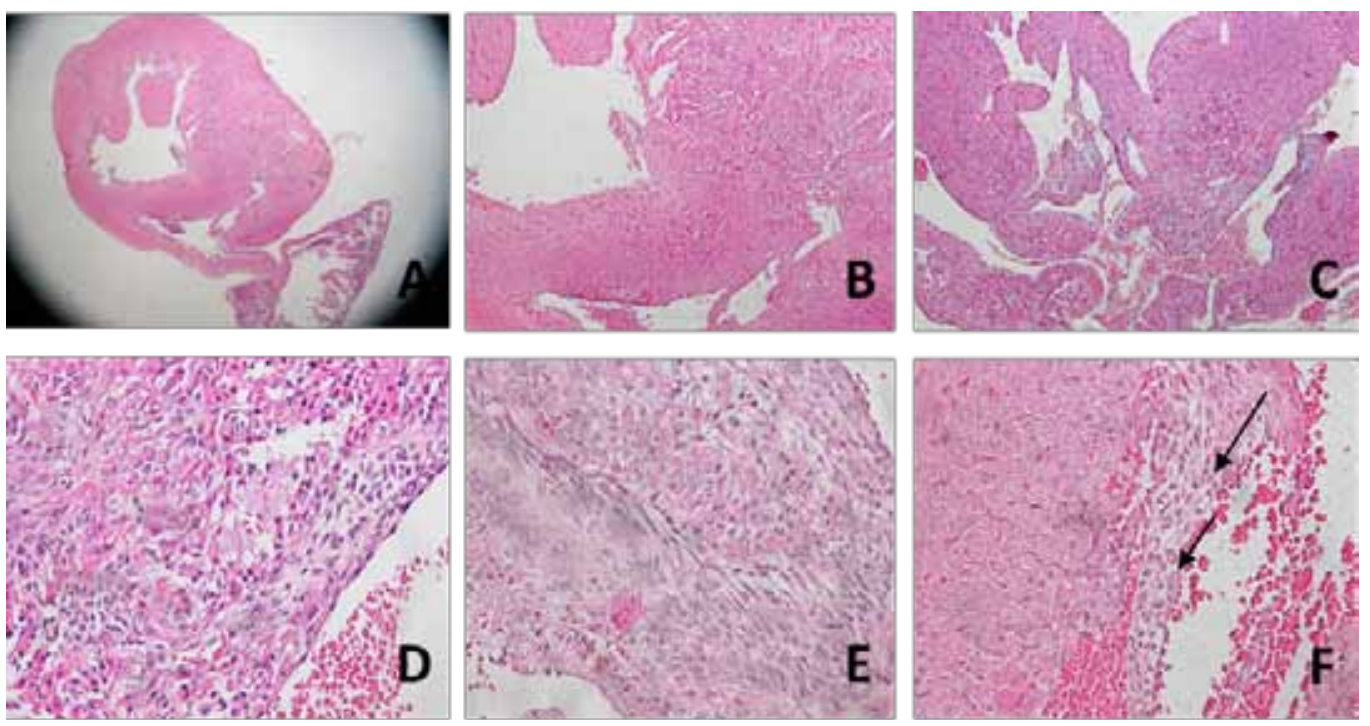

Figura 3. Cortes de miocardio de fetos controles $(A, B)$ y experimentales $(C, D, E, F)$. A) Corte longitudinal de corazón mostrando conformación normal de las cámaras cardíacas (4X). B) Musculatura estriada esquelética conservada del septum interventricular y base del corazón. C) Degeneración muscular mixoide del septum interventricular y paredes del ventrículo (10X). E y F) tejido mixoide en las válvulas atrioventriculares de ambos lados, donde se aprecian células más pequeñas con citoplasma claro y basófilo, así como núcleos reducidos de tamaño. H\&E. 
tratadas fue mayor, el peso promedio de las mismas fue menor que el cuantificado para las crías de las hembras no tratadas. Esta situación explica porqué las hembras tratadas no tuvieron menor peso al compararla con las no tratadas. Similar reducción del peso de fetos por efecto de tiabendazol, flubendazol y mebendazol fue reportada por otros autores en ratas y ratones ${ }^{3,14,23}$.

Un estudio realizado en ratas tratadas con albendazol los días 9 y 11 de la preñez, a una dosis diaria de 5 $\mathrm{mg} / \mathrm{kg}$ de peso causó retardo del desarrollo fetal, caracterizado por reducción de peso corporal y peso de la placenta, así como acortamiento del cordón umbilical y alteraciones estructurales hepáticas, reflejadas en hepatocitos con degeneración hidrópica, citoplasma granular, núcleos de menor tamaño, sinusoides dilatados y linfocitos numerosos. Los fetos tratados, además de las malformaciones, mostraron aspectos que sugirieron inmadurez al ser comparados con los controles ${ }^{13}$.

En el presente estudio, la disminución del tamaño del corazón de las crías experimentales se vinculó con evidencias de degeneración mixoide del miocardio, producto de la acumulación intersticial de mucopolisacáridos que causan alteraciones de la matriz extracelular y daño de las fibras musculares cardíacas ${ }^{17}$. Tales daños deberían atribuirse al efecto vasoconstrictor del albendazol sobre el flujo sanguíneo placentario ${ }^{7}$, alteración que al reducir el aporte sanguíneo habría provocado menor proliferación celular y grados variables de alteraciones degenerativas, que resultarían en alteración funcional ${ }^{16}$. Placentas con flujo sanguíneo disminuido causan hipoxia fetal significativa, la cual cursa con retardo de crecimiento intrauterino y disminución del tamaño de los órganos internos, incluyendo el corazón ${ }^{10}$.

Los efectos teratogénicos de albendazol fueron reportados en diferentes especies animales, en las que provocaron anoftalmia, microftalmia, atresia anal, rabo corto, retorcido o ausente, labio leporino, paladar hendido, hidronefrosis y espina bífida 9, 13,22 . Coincidentemente, en esta investigación se evidenciaron agenesias de rabo y mortalidad fetal intrauterina. Se afirma que las malformaciones podrían ser causadas por la adición de la droga a macromoléculas de los tejidos ${ }^{13}$. A su vez, la malformación podría conducir a un grado excesivo de mortalidad celular en los tejidos embrionarios ${ }^{22}$.

En conclusión, los cambios observados en las crías de madres tratadas con albendazol fueron caracterizados por la reducción de peso corporal, malformaciones como agenesia del rabo y reducción del tamaño del corazón, así como alteraciones degenerativas del músculo cardíaco, circunstancias a tener en cuenta al administrar esta droga durante la gestación.

Agradecimientos. A la Dra. Victoria Colmenarez, del Laboratorio de Anatomía Patológica del Hospital Veterinario "Dr. Humberto Ramírez Daza" de la Facultad de Ciencias Veterinarias-UCLA (Barquisimeto, Venezuela), por la realización del diagnóstico histopatológico de las muestras, así como al Sr. Ronald Linarez y al Medico Veterinario Jesús Sánchez por su apoyo en el manejo de los animales.

\section{REFERENCIAS}

1. Bombonato KF, Prata GV, Brentegani LG, Sala MA, Vale AS, Lopes RA. 1994. Estudo morfológico de fetos de ratas injetadas com mebendazol. Rev Reg Ciênc 3: 19-24.

2. Delatour P, Parish RC, Gyurik RJ. 1981. Albendazole: a comparison of relay embryo toxicity of individual metabolites. Ann Res Vét 12: 159-67.

3. Delatour P, Garnier F, Benoit E, Longin CH. 1984. Correlation of toxicity of albendazole and oxfendazole with their free metabolites and bound residues. J Vet Pharmacol Ther 7: 139-145.

4. Gabrielli AF, Montresor A, Chitsulo L, Engels D, Savioli L. 2011. Preventive chemotherapy in human helminthiasis: theoretical and operational aspects. Trans $R$ Soc Trop Med Hyg 105: 683-693.

5. Harhay MO, Horton J, Olliaro PL. 2010. Epidemiology and control of human gastrointestinal parasites in children. Expert Rev Anti Infect Ther 8: 219-234.

6. Hotez PJ, Fenwick A, Savioli L, Molyneux DH. 2009. Rescuing the bottom billion through control of neglected tropical diseases. Lancet 373: 1570-1575.

7. Kirkinem, P, Jouppila P, Koivula A, Vuori J, Puukka M. 1983. The effect of caffeine on placental and fetal blood flow in human pregnancy. Am J Obstet Gynecol 147: 939-942.

8. Lanusse CE, Pritchard RK. 1993. Relationship between pharmacological properties and clinical efficacy of ruminant anthelmintics. Vet Parasitol 49: 123-158.

9. Lapras M, Delatour P, Deschanel J, Lourge G, Camps D, Regnier B. 1973. Étude expérimentale de l'activité tératogéne du parbendazole chez le rat. Bull Soc Sci Vét Méd Comp 75: 117-130.

10. Longo L, Hewitt CW, Lorijn RH, Gilbert RD. 1978. To what extent does maternal exercise affect fetal oxygenation and uterine blood flow? Fed Proc 37: 905.

11. McKellar G, Scott E. 1990. The benzimidazole anthelmintic agents: a review. J Vet Pharmacol Ther 13: 223-247.

12. Moon TD, Oberhelman RA. 2005. Antiparasitic therapy in children. Pediatr Clin North Am 52: 917-948.

13. Moreti DL, Lopes RA, Vinha D, Sala MA, Semprini M, Friedrichi C. 2005. Efectos del albendazol en el hígado de feto de rata. Estudios morfológico y morfométrico. Int. J. Morphol 23: 111-120.

14. Ogata A, Ando H, Kubo Y, Hiraga K. 1984. Teratogenicity of thiabendazole in ICR mice. Fed Chem Toxicol 22: 509-520.

15. Richard-Lenoble D, Chandernier J, Duong TH. 2003. Anti- parasitic treatments in pregnant women and in children in 2003. Med Trop 63: 491-497.

16. Ross CP, Persaud TV. 1986. Early embryonic development in the rat following in utero exposure to alcohol and caffeine. Histol Histopathol 1: 13-17.

17. Smith HA, Jones TC. 1961. Veterinary Pathology, $2^{\circ}$ ed., Lea \& Febiger, Philadelphia, $1061 \mathrm{p}$.

18. Solano L, Acuña I, Barón MA, Morón A, Sánchez A. 2008. Influencia de las parasitosis intestinales y otros an- 
tecedentes infecciosos sobre el estado nutricional antropométrico de niños en situación de pobreza. Parasitol Latinoam 63: 12-19.

19. Teruel MT, Felipe AE, Solana HD, Sallovitz JM, Lanusse CE. 2003. Placental and fetal toxicity of albendazole sulphoxide in Wistar rats. Vet Human Toxicol 45: 131-136.

20. Van den Enden E. 2009. Pharmacotherapy of helminth infection. Expert Opin Pharmacother 10: 435-451.

21. World Health Organization. 2011. Helminth control in school-age children: A guide for managers of control programmes, 2nd ed, Geneva, 90 p. On line: http://whqlibdoc. who.int/publications/2011/9789241548267_eng.pdf
22. Yoneyama M, Ogata A, Hiraga K. 1985. Irreversible in vivo binding of thiabendazole to macromolecules in pregnant mice and its relation to teratogenicity. Food Chem Toxicol 23: 733-736.

23. Yoshimura H. 1987. Teratogenicity of flubendazole in rats. Toxicology 44: 133-136.

\section{SJR Journal \& Country Rank}

\section{Powered by}

\section{Revista Veterinaria aumentó su índice de impacto}

Noticias de Scimago Research Group (Scimago Journals \& Country Ranks, Scopus-Elsevier) comunican que la publicación de nuestra casa, Revista Veterinaria, aumentó su índice de impacto. El índice SJR mide la influencia científica (impacto) del artículo de una revista, expresando cuán importante es el "artículo promedio" de la publicación en la discusión científica global (sistema Thomson Reuters).

Para nuestra revista, tal indicador había sido de 0,03 entre 2008 y 2011, aumentando a 0,05 en 2012 y a 0,11 en 2013, último período evaluado. El aumento fue del $370 \%$. Asimismo, surge para nuestra publicación un sostenido descenso del indicador que relaciona "citas versus autocitas", demostrando que los autores de los artículos están abandonando la práctica de citar sus propias publicaciones anteriores.

Por último, se advierte que según este portal, nuestra "Revista Veterinaria" continúa siendo la única publicación de esta rama de la ciencia que posee índice de impacto en Argentina. Para el resto del cono sur tal distinción recae en Brasil, Chile, Colombia y Venezuela. No registran índice de impacto las revistas de veterinaria de Bolivia, Paraguay, Uruguay, Perú y Ecuador. En revistas de veterinaria, a nivel mundial el mayor índice de impacto (1,22 puntos) recae en Veterinary Microbiology (Amsterdam, Holanda). 